\title{
Underwater endoscopic submucosal dissection for a duodenal neuroendocrine tumor using pocket creation and ring-shaped thread countertraction methods
}

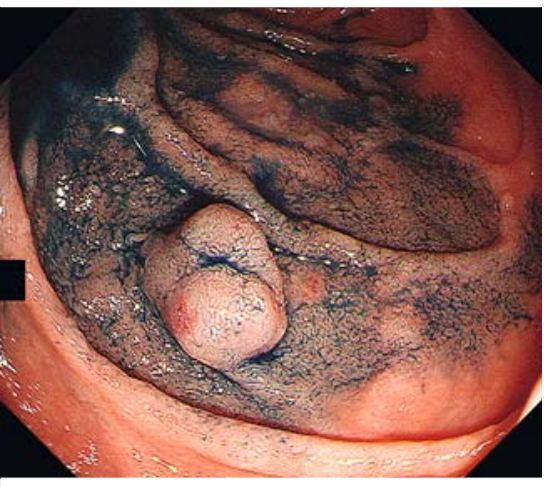

> Fig. 1 Endoscopic image showing a duodenal neuroendocrine tumor at the superior duodenal angle.

A 55-year-old man was previously diagnosed with a neuroendocrine tumor (NET) following biopsy of a duodenal submucosal tumor. He was referred to our hospital for treatment of the lesion. Esophagogastroduodenoscopy revealed a submucosal tumor measuring $9 \mathrm{~mm}$ in diameter at the superior duodenal angle ( $\triangleright$ Fig. 1 a). Endoscopic ultrasound (EUS) revealed a uniform, low echogenic tumor of $8.6 \mathrm{~mm}$ in the submucosal layer. Underwater endoscopic submucosal dissection (UESD) [1] using the pocket creation method (PCM) [2] and ring-shaped thread countertraction technique [3] was performed ( $\vee$ Fig. 2 ; $\vee$ Video 1 ).

First, hyaluronic acid was injected into the submucosa around the lesion, and an incision of about $1.5 \mathrm{~cm}$ was made in the mucosa on the oral side of the lesion. The submucosa below the lesion was dissected using the water-immersion PCM. After a circumferential mucosal incision had been created around the lesion, a $10-\mathrm{mm}$ ring thread was attached to the oral side of the lesion by an endoclip. The other end of the ring thread was then attached, by a second endoclip, to the normal mucosa opposite the lesion ( $\bullet$ Fig.3a). The lesion was completely resected en bloc with

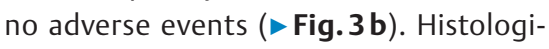

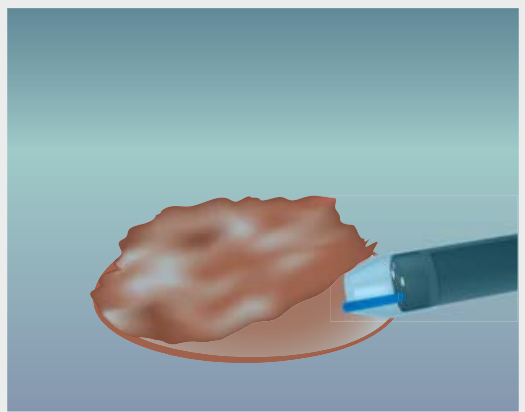

a

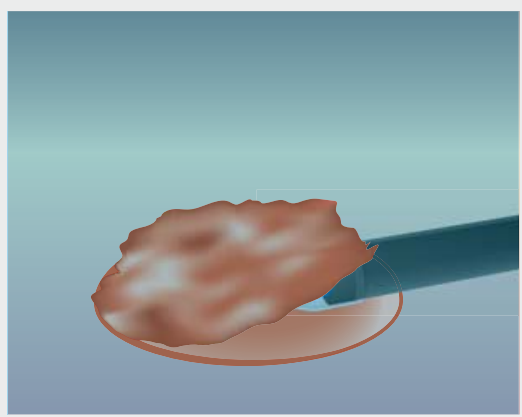
b
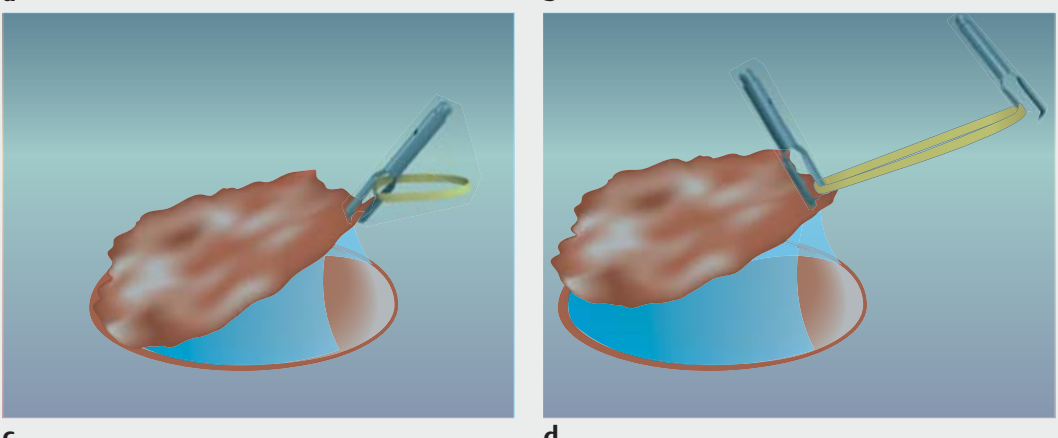

- Fig. 2 Schematic of the steps involved in the ring-shaped thread countertraction technique: a, b underwater endoscopic submucosal dissection using the pocket creation method; c attachment of one end of the 10-mm ring thread to the oral side of the lesion by an endoclip; $\mathbf{d}$ attachment of the other end of the ring thread to the normal mucosa opposite to the lesion by the clip, which allows sufficient traction to be obtained with the ring-shaped thread.
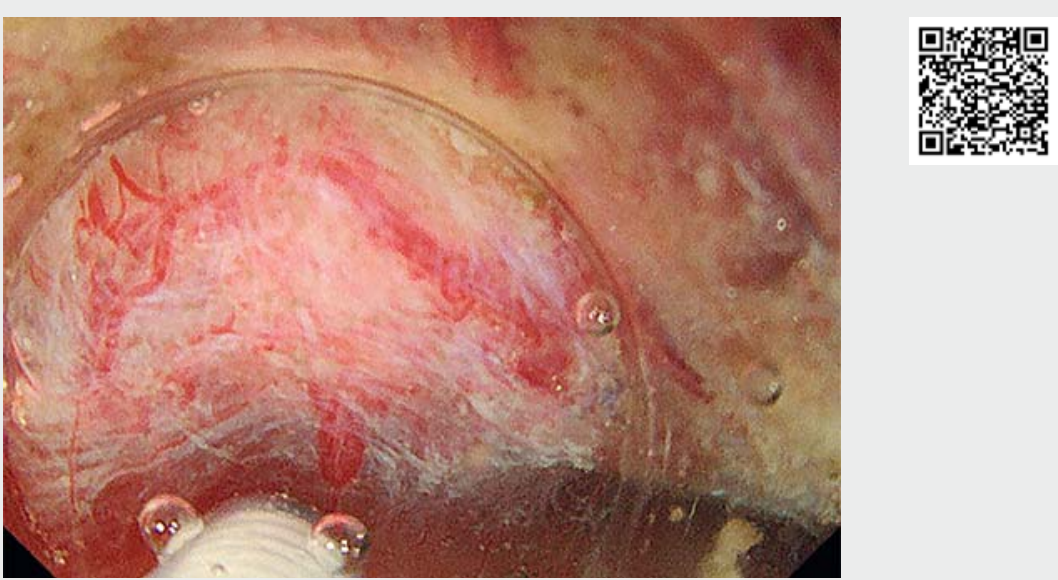

Video 1 Underwater endoscopic submucosal dissection of a duodenal neuroendocrine tumor using the pocket creation method and the ring-shaped thread countertraction technique. 


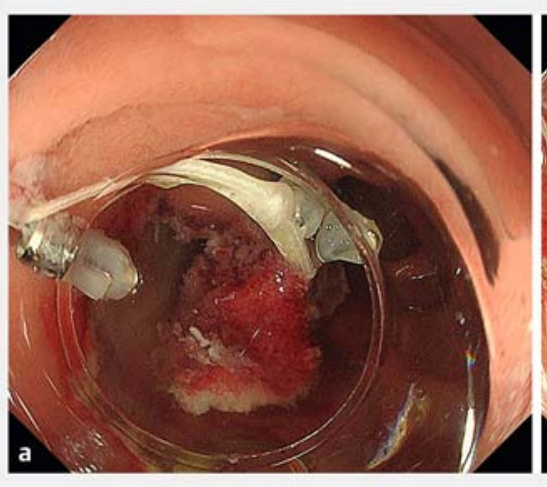

- Fig. 3 Endoscopic images showing: a the ring-shaped thread countertraction technique, with one of the clips attached to the oral side of the lesion and the other to the duodenal wal opposite the lesion; $\mathbf{b}$ the duodenal ulcer after successful endoscopic submucosal dissection.

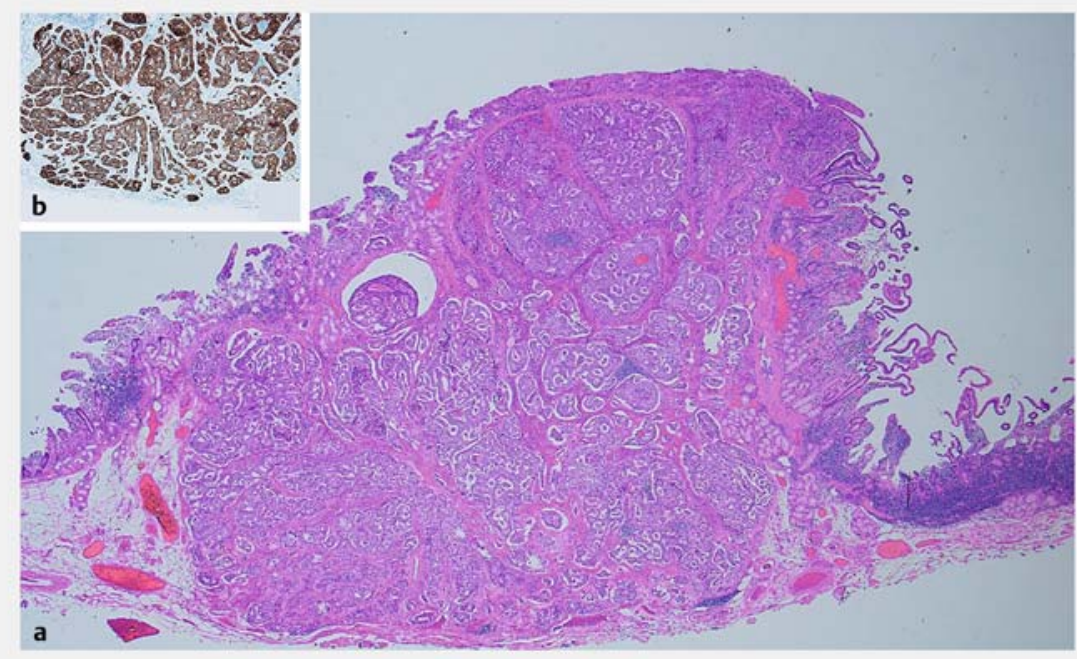

- Fig. 4 Histopathological view of the en bloc resected neuroendocrine tumor: a on hematoxylin and eosin (H\&E) staining; b showing positive staining with chromogranin A.

cal examination confirmed a gastrointestinal NET G1 with submucosal invasion $(2460 \mu \mathrm{m})$ ( $\mathbf{F i g . 4})$. The vertical and horizontal margins were negative for tumor.

Duodenal NETs are rare; they represent $3.8 \%$ of all gastrointestinal NETs [4]. Endoscopic treatment may be performed for duodenal NETs measuring $\leq 10 \mathrm{~mm}$ in diameter and confined to the submucosal layer, without metastasis.

Common endoscopic treatments for duodenal NETs have included endoscopic submucosal resection with a ligation device, endoscopic mucosal resection

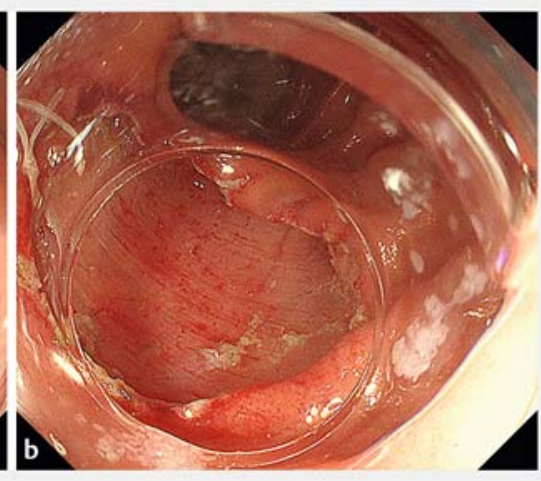

\section{Competing interests}

The authors declare that they have no conflict of interest.

The authors

Mitsuhiro Kono, Yasuaki Nagami, Daiki Kitagawa, Taku Manabe, Masaki Ominami, Shusei Fukunaga, Yasuhiro Fujiwara

Department of Gastroenterology, Osaka City University Graduate School of Medicine, Osaka, Japan

\section{Corresponding author}

\section{Yasuaki Nagami, MD}

Department of Gastroenterology, Osaka City University Graduate School of Medicine, 1-4-3, Asahimachi, Abeno-ku, Osaka, 545-8585, Japan

Fax: +81-6-66453813

yasuaki1975@hotmail.com

\section{References}

[1] Nagata M. Underwater endoscopic submucosal dissection in saline solution using a bent-type knife for duodenal tumor. VideoGIE 2018; 22: 375-377

[2] Miura Y, Hayashi Y, Lefor AK et al. The pocket-creation method of ESD for gastric neoplasm. Gastrointest Endosc 2016; 83: 457458

[3] Mori H, Kobara H, Nishiyama $\mathrm{N}$ et al. Novel effective and repeatedly available ringthread counter traction for safer colorectal endoscopic submucosal dissection. Surg Endosc 2017; 31: 3040-3047

[4] Modlin IM, Lye KD, Kidd M. A 5-decade analysis of 13,715 carcinoid tumors. Cancer 2003; 97: 934-959

[5] Oono Y, Shinmura K, Hori K et al. Endoscopic submucosal resection using a ligation device without injection for duodenal neuroendocrine tumors. Surg Endosc 2019; 33: 20082014

\section{Bibliography}

Endoscopy_UCTN_Code_TTT_1AO_2AG

\section{Acknowledgments}

We would like to thank Editage (www.editage. com) for English language editing.
Endoscopy 2021; 53: E110-E111

DOI 10.1055/a-1198-4153

ISSN 0013-726X

published online 13.7.2020

(c) 2020. Thieme. All rights reserved.

Georg Thieme Verlag KG, Rüdigerstraße 14, 70469 Stuttgart, Germany 